\title{
5 Denk- und Sprachstörungen von schizophrenen Kindern
}

\subsection{Klinische Beobachtungen}

Auf einige Besonderheiten sprachlicher Auffälligkeiten bei frühkindlichen Psychosen wurde bereits im Kapitel 4 kurz eingegangen. Wegen der Bedeutung - klinisch-phänomenologisch, neuropsychologisch, differentialtypologisch - wird das Thema eingehender in diesem Kapitel behandelt.

Die Sprache schizophrener Kinder ist durch einen ausgeprägten Verlust an Reichhaltigkeit und Ausdruckskraft gekennzeichnet. Die sprachlichen Äußerungen sind dürftig, schütter und inhaltsarm, und sie zeichnen sich durch eine Neigung zu stereotypen, nichtssagenden Floskelbildungen aus.

Ähnlich wie bei schizophrenen Erwachsenen sind bei kindlichen Schizophrenen eindrucksvolle Auffälligkeiten des Denkens und der Sprache zu beobachten:

\section{Denkstörungen}

- Denkzerfahrenheit, Gedankenabreißen, unverständlicher Sprachinhalt

- assoziative Lockerung, plötzlicher Themenwechsel, Ideenflucht

- verschrobenes Denken

- Vorbeireden

- Konkretismus

Auffälligkeiten der Sprache

- Mutismus

- Wortkargheit

- Logorrhoe, Rededrang 
- Stereotypien

- Echolalie

- Paralalie, Stammeln

- monotones, modulationsarmes Sprechen

- Dysprosodie

- Clanging, es werden klangähnliche anstelle von bedeutungsangemessenen Wörtern benutzt

- hastiges Sprechen

- Sprechen in Halbsätzen

- Paragrammatismen, Paraphrasien

- a-logische, exzentrische Sprache

- mangelnde bzw. keine Verwendung von Konjunktionen

- mangelnde bzw. keine Verwendung von Kohäsionen

- Neologismen

Es gibt nur wenig systematische linguistische Studien über Sprachauffälligkeiten bei schizophrenen Kindern. Es gibt aber, vor allem in der älteren Literatur, sehr schöne Beschreibungen von Sprachauffälligkeiten bei solchen Kindern. Allerdings haben Sprachauffälligkeiten bei schizophrenen Kindern nicht das gleiche Interesse gefunden wie Sprachstörungen beim frühkindlichen Autismus Kanner.

Die Arbeitsgruppe um R. Caplan hat sich eingehend mit den sprachlichen Besonderheiten schizophrener Kinder beschäftigt und in Anlehnung an die Scale for the Assessment of Thought, Language and Communication (TLC) von Andreasen (1979) die Kiddie Formal Thought Disorder Rating Scale entwickelt. Mit diesem Instrument ist es möglich, Sprach- und Denkstörungen bei kindlichen Schizophrenen herauszufiltern, speziell eine Lockerung der Assoziationen und a-logisches Denken. Caplan et al. (1992) kamen zu dem Ergebnis, dass schizophrene Kinder ähnliche Sprechstörungen aufweisen wie schizophrene Erwachsene. Ihre Sprache sei durch 3 Charakteristika gekennzeichnet:

- eine Beeinträchtigung beim Herstellen von logischen, gedanklichen bzw. sprachlichen Verknüpfungen durch Zusammenfügen kontextrelevanter Sätze bzw. Satzteile (Konjunktionen)

- eine verminderte lexikale Kohäsion, Bedeutungszusammenhänge gehen verloren

- Neigung zu Auslassungen (ellipsis), es werden Wörter und Satzteile ausgelassen, welche eine Sinnbeziehung zum zuvor Gesagten herstellen

Die Sprach- und Sprecheigentümlichkeiten der Sprache bei schizophrenen Kindern (VEOS, Erkrankungsbeginn $\leq 13$ Jahre) sind sehr vielfältig und uneinheitlich: Mutismus, Rededrang, teilweise beides bei ein und demselben Kind. Manche schizophrene Kinder zeigen einen unmotivierten Wechsel der Stimmstärke mit lautem oder leisem VorSich-Hinreden. Die Äußerungen erscheinen unmotiviert und zusammenhangslos, teilweise mit phantastischen, unverständlichen Inhalten. Es kommt zu Wortveränderungen, Wortneubildungen (Neologismen), zum Aneinanderreihen scheinbar sinnloser Wörter, zu sinnlosem Wiederholen von Silben oder Wörtern oder Satzbruchstücken (Verbigerationen). Typisch sind manierierte Gestelztheiten oder Verschrobenheiten des sprachlichen Ausdrucks (ein 12-jähriger schizophrener Junge: „Ich bin innerlich so ausgegloist“ - er meinte wohl „aus dem Gleis geraten“ - engl. derailment). 
Atschkova (1966) berichtet, dass bei schizophrenen Kindern ohne ersichtlichen Grund der Sprachgebrauch ausbleibe, dass manche Kinder im Flüsterton Selbstgespräche führen, aber Fragen nicht beantworten und keinen Gesprächskontakt mit den Angehörigen haben. Kudrjawzewa (1967) beobachtete bei den von ihr untersuchten schizophrenen Kindern, Neologismen, Reimereien, Danebenreden und inhaltlose Reden über technische Themen. Sie beschreibt Monotonie und Armut in der Sprache bei diesen Kindern. Das Kind benutze die Sprache selten als ein Kommunikationsmittel. Manche Autoren berichten vom Sprech- und Rededrang schizophrener Kinder (Kudrjawzewa 1967, v. Stockert 1956, Stutte 1963). Dieser zeige sich in ,fruchtloser Klügelei, in Vorbeireden oder Wiederholungen von Fragen“" (Kudrjawzewa 1967).

Manche Patienten formulieren recht originelle Wortspiele. So sagte ein Patient: „Ich dacht', ich wär' ein Champion und werd' doch nur ein Champignon." Die darin sich zeigende Fähigkeit zur Selbstironie und zum selbstkritischen Wortspiel ist durchaus typisch für das Denken Schizophrener und für deren Kreativität.

Eine der ältesten Beschreibungen der Sprache bei kindlichen Schizophrenien stammt von L. Voigt aus dem Jahre 1919. Bei einem 10-jährigen von ihm beschriebenen Mädchen zeigt sich auch der Verlauf einer psychotischen Sprachstörung. Als das Mädchen das Vaterunser aufsagen soll, sagte es erst: „Das darf man nicht“, fängt dann aber an „Vater, Vater, Vater unser, unser, unser, unser, im, im, im, im, Himmel, Himmel, Himmel, Himmel ...“. Auch beim Lesen wiederholt es jedes Wort mehrmals, liest aber sonst richtig. 3 Jahre später, im Alter von 13 Jahren, klingt die Sprache sehr abgehackt, das Mädchen artikuliert überdeutlich, monoton, langsam, wie auswendig gelernt, es spricht mit scharfer Betonung der Konsonanten, Wort für Wort ist scharf abgegrenzt. Manche sprachliche Äußerungen sind zwischendurch fließend, mit normaler, natürlicher Betonung und Sprachmelodik. Inhaltlich ist die Sprache verarmt, beschränkt sich auf wenige kurze Sätze, wie „will brav sein, Verzeihung, will folgen“, ,kann schreiben“, „kann lesen“, ,,schön lesen“. Im Alter von 14 Jahren liest das Mädchen fließend mit natürlichem Wortklang, mit Verständnis für Sinn und Inhalt der Sätze. Im Alter von 15 Jahren wiederum Verbigerationen, das Mädchen wiederholt stereotyp:

„Ich will brav sein, ich will folgen, ich will es noch einmal versuchen.“

\subsection{Charakteristika sprachlicher Auffälligkeiten bei schizophrenen Kindern}

Die Sprache schizophrener Kinder wird oft als „, desintegrative Sprache“ bezeichnet. Hinter diesem Begriff verbirgt sich eine ganze Reihe von linguistischen Phänomenen wie:

\subsubsection{Veränderungen des Sprechrhythmus und der Sprechgeschwindigkeit}

Die Veränderungen des Sprechrhythmus und der Sprechgeschwindigkeit zeigen sich in einer abgehackten Sprache, abnormen Betonungen von Konsonanten im Vergleich zu Vokalen, unüblichen Satzbetonungen, raschem Herunterleiern oder im Gegenteil in abnormen Überdehnungen von Wörtern und Sätzen. 


\subsubsection{Veränderungen der Sprachmelodie (Dysprosodie)}

Schizophrene Kinder neigen zu singender Sprechweise, mehr oder weniger melodisch, meistens in abnormer Tonhöhe. Häufig verfallen die Kinder in ein singsangartiges Leiern, ein monotones Vorsichhinbrummen, oder sie wiederholen stereotyp, litaneiartig irgendwelche Melodien. Ein 1o-jähriger schizophrener Junge spielte dabei „Orchester“ und sprach in diesem Zusammenhang von „Hermann“ (das Hermann-Hagestedt-Rundfunkorchester damit meinend - Eggers 1973, S. 66).

Typisch sind auch sinnlose Reimereien, wie bei einem 14-jährigen Jungen:

„Karl May ist vorbei, ach armes, deutsches Mägdelein, es kann ja nimmer sein ... Ströme inhaliert, doch nicht imponiert ... es ist vorbei, ich bin wie Brei, Gelenke gehören dein, die rechte ist nicht mein, es ist mir einerlei, der Tod, er eilt vorbei“ (Voigt 1919, S. 193).

\subsubsection{Wiederholen von Silben, Wörtern und Satzteilen (Verbigerationen)}

Nachfolgend ein Beispiel für Verbigerationen und sog. Wörterketten (,word chaining“) bei einem 53-jährigen Patienten, der im Alter von 10 Jahren akut an einer paranoidhalluzinatorischen Schizophrenie erkrankt war. Dieser Patient ist seit 30 Jahren hospitalisiert und antwortete mir bei der Nachuntersuchung auf die Frage, ob die Stimmen ihm etwas sagen würden:

„Ach so, ins Ohr rein, was erzählen, vom Fernsehen wasins Ohr rein, noch eintausendsiebenhunderte Jahre, zehn mal, tausend, tausend, tausend, tausend, eintausend, ja ja, also, ach.....

Auf meine Frage, was ihn von anderen Menschen unterscheide, antwortete er mir:

„Das Richtergeben, das hab’ ich nicht gleich raus gehabt, du lieber Cott, ... die sind alle abgehauen, der frisst uns auf bleib hier, bleib hier, bleib hier, erstmal nicht, erst das Mädchen und ich möcht's nicht zeigen, schimpfen, Schwein, ich kann's nicht mehr sprechen, zeig' doch, ich zeig's ja auch“. Er lacht und klatscht dabei in die Hände. (Frage: Ist es also gefährlich?) Antwort: „Was?“ (Frage: Dass da jemand Sie auffrisst?) Antwort: „Ach so, ja, wenn der wiederkommt auf die Welt.“ (Frage: Wer kommt denn wieder?) Antwort: „Ich komm' wieder auf die Welt, ich komm' wieder, das Unkraut vergisst sich nicht, das kommt immer wieder, Unkraut, Unkraut, Jacobs Krönung, Unkraut schmeckt auch.“

Beim Sprichwörterdeuten assoziiert der Patient zu dem Sprichwort „Der Apfel fällt nicht weit vom Stamm“:

„Wenn der Apfel auf den Ast fällt, dann wird der Baum zum lieben Gott, dann fällt der Ast, der Apfel lässt sich vom Igel fressen. Der Apfel hat Angst der Baum wird der richtige Cott werden. Der Baum hält den Apfel fest, am Zweig. Bringt Kinder auf die Welt, der Apfelbaum. Es müsste mehr Bäume geben, der Apfelbaum reicht nicht aus, Tomate, Johannisbeeren, Himbeeren, Erdbeeren, Stachelbeeren ....”.

Der letzte Satz ist ein Beispiel dafür, dass Früchtenamen kettenförmig aneinander gereiht werden („object chaining“). Eine ausführliche Darstellung der gesamten Kran- 
kengeschichte vom 10. Lebensjahr bis zum Alter von 53 Jahren findet sich im Anhang (Kap. 20, Nr. 4.4.1.6b).

Einen ganz anderen Charakter hat das Wiederholen von klangähnlichen Buchstaben, Silben und Wörtern in Form von Alliterationen, welche v.a. die altgermanische Dichtung kennzeichnet (Roland der Riese vorm Rathaus zu Bremen“). Es handelt sich dabei um ein poetisches Stilmittel in Form von Wiederholungen gleich klingender Anlaute in betonten Silben ${ }^{1}$.

Nachfolgend zwei schöne Beispiele für solche gekonnt künstlerisch gestaltete Alliterationen eines im Alter von 11 Jahren an einer schleichend beginnenden kindlichen Schizophrenie erkrankten Patienten, die er im Alter von 27 bzw. 30 Jahren verfasst und dem Verfasser zugeschickt hat:

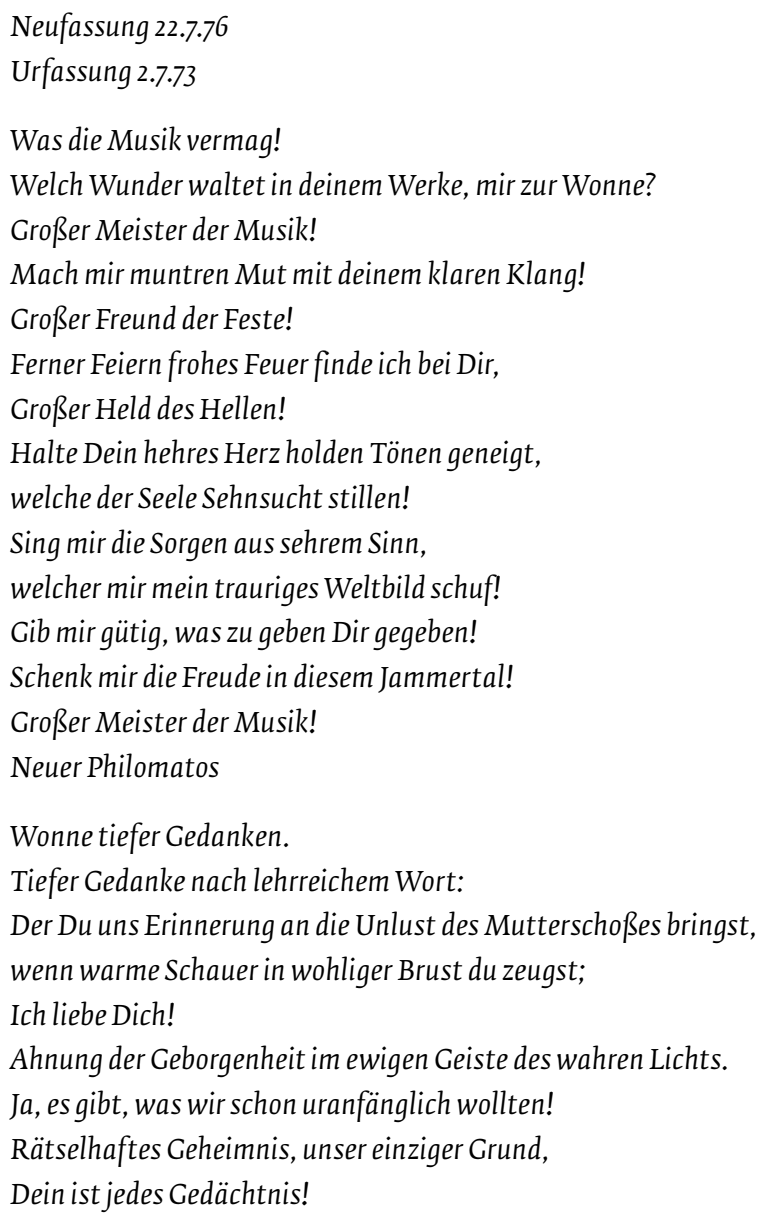

Beiden Gedichten eignet durchaus ein Hang zum Pathetisch-Floskelhaften, aber man spürt die Sehnsucht des Patienten nach Trost und Geborgenheit, welche einem Ge-

1 Duden Band 8: Sinn- und sachverwandte Wörter und Wendungen. Mannheim-Wien-Zürich: Biographisches Institut. Dudenverlag 1972. 
fühl der krankheitsbedingten Bedrohtheit und Verlassenheit entspringen mag. In einem Brief, den er als 31-Jähriger schrieb, schrieb er denn auch: „Ich brauche Ruhe und Geborgenheit in Geistigkeit und Geistlichkeit.“

Die Psychose des Patienten begann im Alter von 11 Jahren mit Widersetzlichkeit, sozialem Rückzug, uneinfühlbaren zwanghaften Ritualbildungen, in die er auch die Eltern einbezog, zunehmender Verschrobenheit, Negativismus, Nahrungsverweigerung, Gang- und Sprechauffälligkeiten (gestelzt-manierierte Sprache, marionettenhaft-steifer Gang) sowie paranoiden Wahnideen (z.B. das Essen sei durch Strahlen aus dem Fernsehapparat vergiftet) - Ausführliche Falldarstellung an anderer Stelle (s. Kap. 4.4). Das erste Gedicht unterschrieb der Patient mit „Neuro Philomatos“, damit spielte er auf seine Existenz als eine Art „Privatgelehrter“ an: Seit seinem Abitur und einigen Semestern an der Universität zog er sich völlig in das Haus seiner Eltern zurück und beschäftigte sich als „Sprachwissenschaftler“ bzw. als „Philomat“(Philomathie (gr.) = Wissensdrang) mit Anglistik, Romanistik, Slawistik und Orientalistik sowie mit Theologie, Musik, Medizin, Philosophie, Psychologie, Soziologie, Politik, Wirtschaft, Botanik ...

\subsubsection{Assoziative Intrusionen}

Dabei handelt es sich um das Einfließen unsinniger Wörter, die nur in sehr lockerer assoziativer Beziehung zu bestimmten Satzelementen des Gesprochenen stehen. So assoziiert der in Kapitel 5.2.3 zitierte Patient (Kap. 20, Nr. 4.4.1.6b) sein eigenes Wiederkommen mit dem Wiederkommen von Unkraut und dies geht über in assoziative Silbenwiederholungen („Ich komm' wieder aufdie Welt, ich komm' wieder, das Unkraut vergisst sich nicht, das kommt immer wieder, Unkraut, Unkraut, Jacobs Krönung, Unkraut schmeckt auch.“)

Assoziationen müssen immer wieder auf ihren Sinn hin überprüft werden. Misslingt dies, kommt es zu unkontrollierten, irrelevanten Assoziationen. Vieles spricht dafür, dass bei schizophrenen Patienten der Prozess des kontrollierenden Planens, Vergleichens und Überprüfens beeinträchtigt ist. Neurobiologisch ist diese Fähigkeit auf der subkortikalen Ebene u.a. an ein intaktes Zusammenspiel des hippocampal-amygdaloidalen Neuronenkreises und auf der kortikalen Ebene u.a. an das geordnete Funktionieren des dorsolateralen präfrontalen Cortex (DLPFC) mit dem Sitz des sog. Arbeitsgedächtnisses und seiner Verschaltungen mit anderen Kortexregionen geknüpft (vgl. auch Kap. 5.8, 12.3 u. 12.4).

\subsubsection{Paragrammatismen}

Als Paragrammatismen werden verschiedene Sprachstörungen zusammengefasst, welche bei schizophrenen Kindern und Erwachsenen als „Schizophrasie“ bezeichnet werden. Die Sprache ist durch abnorme Satzkonstruktionen, ungewöhnliche Verknüpfungen von Wörtern und Phonemen, Alterationen lexikaler Phoneme, inadäquate Verknüpfungen lexikaler Morpheme und Affixen oder durch Verbigerationen gekennzeichnet. Syntaktische Regeln werden nicht beachtet. Daraus resultiert eine Unfähigkeit, phonetische Elemente in einen korrekten semantischen Zusammenhang zu integrieren und Morpheme in adäquater Weise zu Wörtern und schließlich zu Sätzen zusammen zu setzten. Diese Störungen können schließlich zu einem regelrechten „Wort-Salat“ führen. 
Ein typisches Beispiel für ungewöhnliche, unkonventionelle Wortkombinationen (semantische und phonemische Paraphrasien) wird aus den Äußerungen eines 15-jährigen Jungen deutlich, der im Alter von 11 Jahren an einer schleichend beginnenden Schizophrenie erkrankte: Er klagte darüber, dass „die Gedanken von mir übertragen werden“, von wem wisse er nicht, es sei so eine Art „Gedankenleitwerk“. Ähnliche Beispiele: „Zeitgefäß“ anstelle von Uhr, „Nahrungsgefäß“ anstelle von Magen.

\subsubsection{Abgeleitete oder zusammengesetzte morphemische Paraphrasien, Neologismen}

Weitere typische Sprachstörungen sind sog. abgeleitete oder zusammengesetzte morphemische Paraphrasien: Lexikale Morpheme werden inadäquat mit einem Affix (abgeleitete morphemische Paraphrasie) oder mit einem anderen Morphem (zusammengesetzte morphemische Paraphrasie) verknüpft (Lecours 1993). Daraus resultieren ungewöhnliche Wortneuschöpfungen (Neologismen) wie z.B. „Erhaltungsunannehmlichkeit“ (= zusammengesetzte morphemische Paraphrasie), „Ernstlichkeit“, „Apothekigkeit“ (= abgeleitete morphemische Paraphrasie). Diese ungewöhnlichen und fremdartigen Wortneuschöpfungen haben zwar einen umgangssprachlichen Ursprung, die verschiedenen lexikalischen Komponenten werden jedoch inkorrekt oder auf unkonventionelle Weise miteinander verknüpft.

Die eben genannten Beispiele für morphemische Paraphrasien stammen ebenfalls von einem im Alter von 13 Jahren akut erkrankten Mädchen, bei dem alle 7 Wochen jeweils 14 Tage lang anhaltende psychotische Phasen mit optischen und akustischen Halluzinationen, Beziehungsideen, Angstzuständen, Wahnstimmung und Schlaflosigkeit mit ängstlich-autistisch-abwehrendem Verhalten auftrat. Zwei Jahre nach der ersten Episode trat eine Verschlimmerung ein, die Patientin stand unter dem Einfluss imperativer akustischer Halluzinationen, die ihr Befehle erteilten, sie litt unter schweren katatonen Erregungszuständen verknüpft mit wahnhaften Bedrohtheitserlebnissen. Und in solch einem Erregungszustand schrie es: „Das Feuer, das Feuer, es brennt, es brennt, es brennt“(Verbigerationen). Das Denken des Mädchens war zerfahren. So antwortete es auf die Frage, ob es sich nicht wohl fühle:

„Ich bin verfolgt von den Menschen. Ich will sterben, ich war schon in Vergasung, aber mein Herz wollte nicht sterben. Sie schlachten die Mädels ab. Ich habe gehört von den Leuten. Erhaltungsunannehmlichkeit. Und mir fällt alles aufdie Augen." (Genaue Falldarstellung des Gesamtverlaufs siehe Kapitel 20, Nr. 4.4.1.4b)

\subsubsection{A-logische, exzentrische, bizarre Sprache}

Schizophrene Kinder können häufig keine kontextbezogenen Antworten geben, sie scheinen internen Gedanken und Inspirationen zu folgen, von denen sie anscheinend irgendwie vollständig gesteuert werden.

Ein 14-jähriges Mädchen antwortete auf die Frage warum es im Bett liege: „Weil ich an die Englein glauben soll. Ich bin ein Backfisch und darum muss ich für die Kinder an die Englein glauben". Auf die Nachfrage, ob das der Grund sei, weshalb es nicht aufstehen könne, antwortete es: „Nein, aber weil der Stuhl da so dicht am Tisch steht. “Auf die Frage, ob es krank 
sei antwortete es: „Sie sagten, wenn sie von der Kirche kamen, mit dem Handschuh, mit dem Schlüssel. "Merkwürdig verschroben erschien auch ihre Antwort auf die Frage, wo es ihr denn am meisten weh tue: „An der Zunge." - [Die Zunge als Symbol für Denken und Sprechen?].

Die Sprache Schizophrener hat einen egozentrischen Charakter analog zur Sprache von Kleinkindern, die wie selbstverständlich davon ausgehen, dass der erwachsene Zuhörer ihr kindliches semantisches Referenzsystem teilt.

\title{
5.2.8 Lockerung der kohäsiven Bezüge, „Entgleisung“ (derailment) des Gedankengangs
}

Die Gedanken verlieren ihre kohäsive Beziehung zueinander. Durch die Störung des formalen Ablaufs der Gedanken kommt es zu deren „Entgleisung“, unabhängig von der Verwandtheit oder der Zusammengehörigkeit der jeweiligen Themen. Die spontansprachlichen Abweichungen beschränken sich nicht nur auf die Grammatik oder das Lexikon, sondern auch auf die Vertextung und die Art und Weise der Gesprächsführung und Gesprächsbeteilung. Die Sprache der Patienten kann sich „,von einem eigentümlichen Bezugsrahmen zu einem anderen verlagern“ (Andreasen 1979).

\begin{abstract}
Bei der Nachuntersuchung einer 30-jährigen Patientin, die im Alter von 12 Jahren erkrankt war, konnte eine „semantische Dissoziation“ ihrer Sprache festgestellt werden. Befragt nach dem Unterschied zwischen Teich und See antwortete sie: „Der Teich ist mit Sand besät, der Fluss ist im Wasser und wenn man das Wasser mit dem Feuer zusammentut, ist es dasselbe - beides ist gefährlich, ich weiß was gemeint ist, aber wie das zusammenhängt, das weiß ich auch nicht." Sie war ebenfalls nicht in der Lage, Definitionen sinngemäß und richtig wiederzugeben. Auf die Bitte, die Bedeutung des Sprichwortes "Morgenstund hat Gold im Mund“ zu erklären antwortete sie: „Morgens darf man nicht singen oder pfeifen, sonst gibt's was Schlechtes zu hören“. Auf die Frage was das Wort "Gold“ bedeute sagte sie: „Von morgens 5 Uhr bis 7 Uhr bedeutet das. " Ausf. Darstellung des Krankheitsverlaufs im Anhang (Kapitel 20, Nr. 4.4.1.6f)
\end{abstract}

In der Antwort der Patientin erkennt man eine Tendenz zum Konkretismus. Ebenso wird eine Missachtung dessen deutlich, was in dem Sprichwort zum Ausdruck gebracht werden soll. Die Patientin bleibt an bestimmten Wörtern hängen und vernachlässigt die übrigen Wörter und Satzteile, die wesentlich für das Verständnis des Gesamtzusammenhangs sind. Die Antworten haben somit den Charakter des Zufälligen, sie sind nicht auf den relevanten Kontext bezogen. Diese Form der kognitiven Störung könnte am ehesten als „semantische Dissozation“ bzw. als „,kognitives Gleiten“ bezeichnet werden.

Auf der neurophysiologischen Ebene spiegelt sich die mangelnde semantische Kontextbezogenheit des Denkens bei schizophrenen Patienten in einer verzögerten Latenz sowie einer verstärkten Negativität der N40o-Welle wider (Nestor et al. 1997). Die Negativität der N40o-Amplitude wird verstärkt durch Sätze mit unsinnigen Satzenden (z.B. die Leute beten in ihrem lokalen Nest statt in ihrer lokalen Kirche) oder durch nicht zusammengehörige Wortpaare (z.B. Arzt-Brot statt Arzt-Krankenschwester). Die N40o ist ein ereigniskorreliertes Potential, welches $400 \mathrm{~ms}$ nach einem sensorischen Stimulus ausgelöst wird. 
In einer experimentellen Studie zum Problemlöseverhalten schizophrener Jugendlicher konnten wir zeigen, dass sie im Vergleich zu neurotischen und gesunden jugendlichen Probanden in einer standardisierten Gesprächssituation signifikant mehr Fokuswechsel vornahmen und schon zu Gesprächsbeginn von der Problem- auf die Beziehungsebene wechselten (Wiemer et al. 1999), was als Ausdruck einer mangelnden Kohäsion zu interpretieren ist, ein Befund, der auch bei erwachsenen Schizophrenen erhoben worden ist (Harrod 1986).

\subsection{Störung des Sprachverständnisses}

Das eben beschriebene Beispiel zeigt, dass nicht nur die expressive Sprache betroffen ist bei schizophrenen Kindern und Erwachsenen, sondern auch die rezeptive Sprache, das heißt das Sprachverständnis. Schizophrene Patienten haben ebenso wie highrisk-for-schizophrenia-children (Kinder schizophrener Eltern) Schwierigkeiten, komplexe grammatikalische Strukturen zu verstehen (Eggers 1991). Diese Störung könnte auf eine Beeinträchtigung der Fähigkeit zurückgeführt werden, eine hierarchische Ordnung und Strukturierung von Wörtern und Sätzen herzustellen, die von Bedeutung für das Verständnis des jeweils Gesprochenen (oder Gelesenen) sind. Das Satzverständnis resultiert nicht aus einem bloßen Zusammenfügen von gesprochenen oder gelesenen Wörtern sondern aus einer logischen Evaluation der Wörter entsprechend ihrer individuellen Bedeutung. Es scheint so, als ob bei schizophrenen Patienten einzelne Wörter und Sätze in Bezug auf ihren Sinngehalt als gleichrangig bewertet werden und eine für das korrekte Verständnis notwendige Hierarchisierung der Bedeutungsrelevanz nicht erfolgt. Daraus könnte die für Schizophrene so typische paralogische, fremdartig-verworrene Sprache resultieren. Beringer (1926) sprach in diesem Zusammenhang von dem „Charakter des Einfallmäßigen, Unverpflichtenden“ schizophrener Äußerungen sowie von „Bedeutungsverschiebungen“, welche er so häufig bei den sprachlichen Äußerungen seiner Patienten beobachtete.

\section{4 linguistische Befunde}

Die Tendenz schizophrener Kinder und Jugendlicher Begriffen und Sätzen den gleichen Stellenwert zuzumessen und eine bedeutungsangemessene Hierarchisierung derselben außer Acht zu lassen, steht in Übereinstimmung mit bereits anfangs zitierten linguistischen Befunden von Caplan et al. (1990). Die Autoren fanden eine signifikante Beziehung zwischen dem Ausmaß a-logischen Denkens und einer verminderten Aufmerksamkeitsspanne. Das Aufmerksamkeitsdefizit und die Tendenz zu a-logischen Denken wurden auf der Basis freier Nacherzählungen einer zuvor erzählten Ceschichte und eines freien Interviews evaluiert. Die assoziative Lockerung zeigte sich in unvorhersehbaren Fokuswechseln in Richtung zu Themen ohne jeden Bezug zum eigentlichen Gesprächsgegenstand, was es dem Zuhörer erschwert, dem Gespräch zu folgen. Caplan et al. (1996) berichten über ähnliche Beobachtungen wie sie Grinker und Holzman (1973) bei schizophrenen Adoleszenten und jungen Erwachsenen gemacht haben: Eine Lockerung der kohäsiven Bezüge und eine Beeinträchtigung der lexikalen Kohäsionen sowie Auslassungen von Wörtern, Sätzen oder Satzfragmenten, die in einem logischen Kontext zu dem zuvor Gesagten stehen. 
Ähnlich wie Caplan u. Mit. bei schizophrenen Kindern beobachteten Docherty et al. (2006) bei schizophrenen Erwachsenen Störungen sowohl der Daueraufmerksamkeit (,sustained attention“) als auch der zielorientierten und planvollen Anordnung und Reihung von Wörtern und Satzfragmenten, kognitive Beeinträchtigungen, die für die Inkohärenz der Sprache und die typischen Denkstörungen Schizophrener verantwortlich zu machen seien.

Eggers (1991) hat auf die Schwierigkeiten hingewiesen, die sowohl schizophrene Kinder und Jugendliche als auch sog. high-risk-for-schizophrenia-children bei der Entwicklung erfolgversprechender Lösungsstrategien zur Bewältigung komplexer Problemlöseaufgaben haben. Wenn man in Anlehnung an Käsermann (1983, S. 137) Sprechen als ein Verhalten konzipiert, welches „zielgerichtet/funktional zur Lösung der Kommunikationsaufgabe (Realisierung der Intentionen) eingesetzt wird“ auffasst, dann kann die Sprachstörung Schizophrener auch als eine Beeinträchtigung der Fähigkeit aufgefasst werden, adäquate Lösungsstrategien zur Bewältigung kommunikativer Probleme zu entwickeln und anzuwenden.

Bei linguistischen Auswertungen von Interviews, Bildbeschreibungen und Nacherzählungen (Leaper \& Emmorey 1985) oder des Kiddie Formal Thought Disorder Spiels (Caplan et al. 1989) konnten Ähnlichkeiten zwischen schizophrenen Kindern und schizophrenen Erwachsenen beobachtet werden. Beide beachteten während ihren Beschreibungen und Erzählungen irrelevante Details und schenkten zuvor Gesagtem wenig Beachtung, es bestand also eine Lockerung der kohäsiven Bezüge, die sprachlichen Äußerungen erschienen infolgedessen fragmentiert und unzusammenhängend bzw. a-logisch.

In einer Untersuchung an schizophrenen Probanden im Alter von 15-41 Jahren fanden Morice u. Ingram (1983) eine Beziehung zwischen der syntaktischen Komplexität der Spontansprache der untersuchten Probanden und dem Erkrankungsalter:

\section{Je geringer die linguistische Komplexität, desto früher der Erkrankungsbeginn.}

Diese Beziehung war unabhängig von der Krankheitsdauer.

Ausgehend von der Beobachtung, dass offensichtlich unangemessene assoziative Einfälle mit geringer oder fehlender Relevanz für den jeweiligen Inhalt bzw. das Thema eines Gesprächs bzw. Interviews oder einer Erzählung in das Sprechen (und Denken) des Patienten „eindringen“ und zum Erscheinungsbild einer assoziativen Lockerung der Sprache bis hin zur Logorrhoe oder gar zum „Wort-Salat“ führen, entwickelten Maher et al. (1993) die Hypothese einer verstärkten Aktivierung oder einer mangelnden Hemmung von gedanklichen Assoziationen bei schizophrenen Patienten.

Ein kohärentes Denken und Sprechen hat zur Voraussetzung, dass störende Einfälle und Gedanken unterdrückt werden. Normalerweise werden nur solche Gedanken zugelassen, die mit dem geplanten Sinn eines Denkinhalts bzw. einer Mitteilung im Einklang stehen. Bei denkgestörten Schizophrenen kommt es dagegen zu inadäquaten, kontextirrelevanten assoziativen Intrusionen in Form von Wiederholungen von Silben, Wörtern und Satzfragmenten („word chaining“), zu Klangassoziationen (,clanging“) oder zu tangentialen oder völlig abwegigen Sätzen oder Satzfragmenten infolge einer unverhältnismäßig starken Aktivierung assoziativer Netzwerke. Dieses psy- 
cholinguistische Phänomen steht neurobiologisch möglicherweise in einem Zusammenhang mit einer Dysfunktion der dopaminergen Transmission. Die Assoziationsstörung schizophrener Patienten kann in sog. Priming-Experimenten untersucht werden (Spitzer et al. 1993).

Das Wort „priming“ = Bahnung, semantische Präaktivierung, steht in folgendem Zusammenhang: Wenn im Rahmen einer Wortentscheidungsaufgabe, bei der möglichst rasch ein Reiz- bzw. Zielwort (target) erkannt werden soll, ein Wort präsentiert wird, was der Bedeutung dieses Zielworts ähnlich ist (sog. prime-word), kommt es zu solch einer semantischen Präaktivierung, die ein rasches Erkennen des Zielwortes ermöglicht. (Beispiel: Birne-Apfel, die Birne wäre das Prime-Wort, der Apfel das Zielwort. Wird das Prime-Wort „Birne“ gezeigt, erfolgt die Erkennung rascher als wenn ein bedeutungsmäßig nicht verwandtes Wort präsentiert wird, wie z.B. „Decke“).

Das Ausmaß des semantic priming gibt an, um wie viel Millisekunden (ms) schneller die Targets bei semantischer Verwandtschaft des Prime-Worts erkannt werden, verglichen mit dem Erkennen des Target-Worts nach Darbietung semantisch nicht verwandter Prime-Wörter.

Bei denkgestörten Schizophrenen kommt es im Vergleich zu nicht denkgestörten Schizophrenen, zu Patienten mit unipolarer Depression und zu Gesunden zu einer vermehrten semantischen Bahnung bzw. Voraktivierung, was Hinweis für eine fehlende Hemmung bzw. verstärkte Aktivierung im Bereich semantischer Netzwerke sein kann. Dadurch könnte das Einstreuen von inkohärenten Assoziationen bzw. Idiosynkrasien (seltene Wörter) erklärt werden - vgl. hierzu Übersichtsarbeit von Maher u. Spitzer (1993).

Man unterscheidet einen automatischen, relativ rasch ablaufenden von einem langsam ablaufenden komplexeren Erkennungsvorgang (recognition process). Letzterer bedeutet, dass bei einem nicht verwandten Reizwort (prime-word) das Erkennen des Zielwortes erschwert ist, und dass ein vergleichsweise größeres lexikales Gedächtnis abgefragt werden muss. Und hierbei spielen die Erwartung (expectancy) und das semantische Erkennen bzw. Wiedererkennen eine Rolle. Letztere Prozesse laufen bei Schizophrenen langsamer ab als bei Gesunden. Neurobiologisch stehen die beschriebenen psycholinguistischen Auffälligkeiten u.a. in einem Zusammenhang mit Störungen fronto-temporaler Funktionen. So wird die Funktion des frontalen Kortex allgemein mit der Planung und Organisation zielgerichteter Verhaltensweisen in Verbindung gebracht. Voraussetzung hierfür ist die Repräsentation von Zielen und Kenntnissen, um diese Ziele verfolgen zu können. Dopamin wirkt ebenso wie Noradrenalin im frontalen zerebralen Kortex als Neuromodulator. Die Rolle des Frontallappens nicht nur für komplexe Problemlöseaufgaben wie den Wisconsin Card Sorting Test sondern auch für die Funktion des semantischen assoziativen Gedächtnisses lässt sich mittels bildgebender Verfahren wie der Positronenemissionstomographie (PET) nachweisen.

Mittels PET und fMRT konnte gezeigt werden, dass der Schweregrad positiver Denkstörungen bei Erwachsenen mit einer Abnahme der neuralen Aktivierung sprachrelevanter Areale im linken superioren temporalen Gyrus und einer Funktionsstörung im anterioren Cingulum korreliert, eine Region, die für die exekutive Kontrolle von Sprache von Bedeutung ist (Kircher et al. 2001, Liddle et al. 2002). 


\subsection{Differentialdiagnostische Hinweise}

Sprachstörungen schizophrener Kinder und Jugendlicher sind durch die ganz unterschiedliche klinische Begleitsymptomatik von manischen und submanischen Episoden im Rahmen einer Bipolar-I- bzw. Bipolar-II-Störung abzugrenzen (Eggers 2011). Logorrhoe (gesteigerter Rededrang) und Ideenflucht (Gedankenjagen) sind typische Symptome im Rahmen (sub-)manischer Episoden, die jedoch oft kurzdauernd sind und einem raschen Phasenwechsel (,rapid cycling“) unterliegen. Insofern sind diese Sprachstörungen im Vergleich zur Logorrhoe bei schizophrenen Psychosen weniger tiefgreifend und erreichen selten das Ausmaß einer typischen Denkzerfahrenheit. Denn die Sprache Schizophrener verfügt über deutlich weniger strukturelle Verknüpfungen als die Sprache von manischen Patienten. Früh beginnende bipolare Störungen können in schizophrene oder schizoaffektive Psychosen übergehen. Kinder mit schizophrenen Psychosen und Kinder mit schizotypen Persönlichkeitsstörungen zeigen ähnliche Denk-, Sprach- und Kommunikationsstörungen (Tompson et al. 1997). Bei schizophrenen Kindern sind jedoch die Schwierigkeiten, lexikale und referentielle Kohäsionen adäquat zu nutzen und sich auf das Wesentliche eines Gesprächs zu fokussieren, stärker ausgeprägt (Caplan et al. 1990, Caplan u. Guthrie 1992).

In einer vergleichenden Untersuchung an Jugendlichen (Alter 12-18 Jahre) mit schizophrenen Psychosen $(\mathrm{n}=20)$, mit einer major depression mit psychotischen Symptomen ( $\mathrm{n}=12)$ und nicht-psychotischen psychiatrischen Erkrankungen $(\mathrm{n}=34)$ sowie einer Kontrollgruppe von Jugendlichen mit internistischen Erkrankungen konnten Makowski et al. (1997) feststellen, dass sich schizophrene Jugendliche hinsichtlich ihrer Denkstörung am deutlichsten von den drei anderen Gruppen unterschieden. Bei den schizophrenen Jugendlichen war eine Neigung zu einem idiosynkratischen, eigenwilligen Sprachgebrauch, zu a-logischem Denken, zu Konfusionen auf der perzeptuellen Ebene, zu assoziativer Lockerung und Inkohärenz zu beobachten, und es bestand eine Unfähigkeit, sich auf eine gestellte Aufgabe realitätsangemessen einzustellen. Das Ausmaß der Denkstörungen wurde mit dem Thought Disorder Index (TDI) bestimmt (Solovay et al. 1986). Die Ergebnisse entsprachen den Befunden einer anderen Studie an Jugendlichen, die mit demselben Instrument untersucht worden waren (Arboleda $u$. Holzman 1985). - Inzwischen ist ein zeitlich weniger aufwändiges Erhebungsinstrument zur Beurteilung auch subtiler formaler Denk- und Sprachstörungen entwickelt worden, der Thought and Language Index (TLI) - Liddle et al. (2002).

\subsection{Prämorbide Sprech- und Sprachstörungen}

Kinder und Jugendliche, die im Erwachsenenalter an einer Schizophrenie erkranken, weisen häufig Störungen ihrer motorischen, sprachlichen und psychosozialen Entwicklung auf (Cannon et al. 1999, Crow et al. 1995, Done et al. 1994, Jones et al. 1994). Bei 33 Kindern mit einer manifesten frühkindlichen Schizophrenie (Erkrankungsalter zwischen 5 und 15 Jahren) fanden Kolvin et al. (1971) in 49\% der Fälle Hinweise auf Entwicklungsverzögerungen, davon häufig Störungen der Sprachentwicklung und der sozialen Adaptation (87\%), andere Autoren berichten über ähnliche Zahlen (u.a. Alaghband-Rad et al. 1995, Hollis 1995, Russell et al. 1989). Unter 49 von Nicolson et al. (2000) untersuchten kindlichen Schizophrenien (Erkrankungsalter 12 Jahre) 
wiesen 55\% Sprech- und Sprachstörungen auf (Störungen der Artikulation und des Sprechrhythmus, Beeinträchtigungen der expressiven und rezeptiven Sprache sowie Störungen der Sprachentwicklung). Ebenso häufig waren prämorbide Störungen der motorischen und sozialen Entwicklung. Kinder mit prämorbiden Sprach- und Sprechstörungen hatten eine stärkere familiäre Belastung mit schizophrenen Spektrumstörungen (Schizophrenie, schizoaffektive Psychosen, schizotypische und paranoide Persönlichkeitsstörungen) und mehr Geburtskomplikationen in der Anamnese.

Häufig werden vor allem von angloamerikanischen Autoren neben prämorbiden Beeinträchtigungen der sprachlichen, motorischen und sozialen Entwicklung auch intellektuelle Behinderungen bei ihren Patienten beschrieben (u.a. Asarnow et al. 1987, 1994, Hollis 1995, Watkins et al. 1988). Da es sich außerdem oft um sehr junge Kinder handelt (Erkrankungsalter vor dem 6./7. Lebensjahr) dürfte es sich bei einem Teil der mitgeteilten Fälle um primär hirnorganisch bedingte psychotiforme Krankheitsbilder mit entsprechenden Störungen der motorischen, sprachlichen, intellektuellen und psychosozialen Entwicklung handeln, nicht aber um echte frühkindliche Schizophrenien. Wir haben jedoch bei unseren 57 durchschnittlich nach 15-jährigem Krankheitsverlauf erstmalig nachuntersuchten Patienten, die im Alter zwischen 7 und 14 Jahren erkrankten, nur vereinzelt Entwicklungsstörungen beobachtet. Bei diesen 57 Patienten handelt es sich um eine Kerngruppe unter strengen Kriterien diagnostizierter und durch Katamnese und Nachuntersuchung verifizierter kindlicher Schizophrenien - von ursprünglich 71 kindlichen Patienten wurden 14 wegen Zweifeln an der ursprünglich gestellten Diagnose ausgesondert. Von den 57 Patienten waren $6(10,5 \%)$ mäßig begabt oder debil - lediglich bei 3 Patienten lag der IQ (HAWIK) unter 80 .

\subsection{Sprachstörung als Störung der Kommunikation}

Sprache steht im Dienste der Kommunikation. Sprache ist ein wesentlicher, wenn auch nicht ausschließlicher Bestandteil unseres Kommunikationsverhaltens, das bei schizophrenen Patienten grundlegend beeinträchtigt ist. So betont Lutz (1938/1939) den Beziehungscharakter der Sprache, und er fasst schizophrene Sprachstörungen entsprechend als „spezielle Beziehungsstörung“ auf.

Bereits lange vor dem Ausbruch einer schizophrenen Psychose können Sprach-, Denkund Kontaktstörungen bei Risikoprobanden (Kinder schizophrener Mütter) beobachtet werden, welche sich von gesunden Kontrollprobanden unterscheiden. Unterscheidungsmerkmale waren: Inkohärenz des Gedankengangs, Lockerung der gedanklichen Assoziationen, Stimmungsinkongruenz des Gesichtsausdrucks und Kontaktschwierigkeiten (Parnas et al. 1982). Somit differenziert auch das präverbale mimische Ausdrucksverhalten Probanden mit einem genetischen Schizophrenierisiko von gesunden Individuen. Die Befunde von Parnas et al. (1982) stehen in Übereinstimmung mit einer anderen highrisk-Studie, bei der sich zeigte, dass die sprachlichen Äußerungen von Kindern schizophrener Eltern durch eine Lockerung gedanklicher und semantischer Bezüge gekennzeichnet waren (Harvey et al. 1982). 


\title{
5.8 Kognitionen ${ }^{2}$
}

Davon ausgehend, dass Sprache als vorgegebene (Bedeutungs-)Struktur des Denkens (Posner 1976) aufgefasst werden kann, können die beschriebenen Denk- und Sprachstörungen als pathologische Ausdrucksformen beeinträchtigter kognitiver Fähigkeiten interpretiert werden, worauf wir im Kapitel 5.4 bereits kurz eingegangen waren. Es gibt ein vorsprachliches Denken, welches Voraussetzung für späteres sprachliches Denken sowohl in der Ontogenese als auch in der Phylogenese ist. So beginnen Kleinkinder etwa mit einem Jahr, Dinge ihrer Umwelt zu benennen. Diese Fähigkeit ist jedoch an kognitive Voraussetzungen geknüpft. Dazu gehören die Fähigkeit zur Objektpermanenz, zu Mittel-Zweck-Analysen und zur Symbolisierung (= Fähigkeit, beliebige Gegenstände als Stellvertreter für etwas anderes zu verwenden, z.B. ein Stück Holz für eine Auto, und damit zu spielen („Symbolspiel“)).

Die moderne Entwicklungspsychologie hat Erkenntnisse von Piaget bestätigt, wonach das Kind bereits über angeborene Erkenntnisstrukturen verfügt, die es ihm erlauben, sich aktiv mit seiner Umwelt auseinandersetzten zu können, wozu neben Wahrnehmungskompetenzen auch „mentale Verarbeitungsmechanismen höherer Art“ gehören könnten (Pauen 2006).

Ganz analog zur Fähigkeit von Schimpansen, außerhalb ihres Käfigs liegende Gegenstände durch das Ineinanderschieben von zwei Stöcken heranzuholen - sie waren sogar in der Lage, nicht passende Holzlatten an deren Enden solange probatorisch durch Nagen zu bearbeiten, bis sie in die Höhlung des anderen Holzes passten - sind auch junge Kleinkinder schon in der Lage, an entfernt liegende Objekte, welche sie begehren, heranzukommen. D.h. sie können, wie Primaten, Problemlösestrategien durch mentales Vorstellen von Probehandlungen entwickeln. Solche präverbalen kognitiven Leistungen bei Tieren (Dohlen, Schimpansen) und beim jungen Kind bezeichnete der Verhaltensforscher O. Koehler als ,unbenanntes Denken“ (O. Koehler 1953). Zu den Schimpansen-Versuchen: W. Köhler 1921.

\begin{abstract}
Allerdings: Im Kleinkindes- und Vorschulalter erfolgt beim Kind normalerweise ein Entwicklungsschub in Richtung abstrakter Konzeptbildungen des Zählens und räumlich-geometrischer Zusammenhänge, die weit über das bei intelligenten Tieren Beobachtbare hinausgehen. Das vorsprachliche Denken bildet jedoch die Voraussetzung für die Befähigung zu sinnvoller verbaler Kommunikation.
\end{abstract}

Der Begriff „Kognition“ umfasst verschiedene mentale Prozesse wie Gedächtnis, Aufmerksamkeit, Wahrnehmung, problemlösendes und planendes Denken. Letztere gehören zu der Gruppe der „Exekutiven Funktionen“, welche bei schizophrenen Patienten sehr häufig in unterschiedlichem Ausmaß beeinträchtigt sind. Exekutive Funktionen sind kognitive Funktionen höherer Ordnung, welche neben der fundamentalen Planung von Handlungssequenzen auch die gesamte Organisation von Handlungsweisen steuern. Dazu gehören die Planung von Partialzielen, das vorübergehende Aufrechterhalten eines Plans und gegebenenfalls dessen Aktualisierungen und Modifikationen. Außerdem beinhalten sie das Monitoring, welche Partialziele erreicht sind

2 Bei der Abfassung dieses Abschnitts war Frau Dipl.-Psych. Ch. Totzeck freundlicherweise behilflich. 
und die Inhibition von (noch) nicht passenden Aktivitäten, sowie die Fehlerentdeckung und Organisation einer adäquaten Reaktion auf Fehler (Reischies 2007). Beeinträchtigungen dieser Handlungsschritte stellen einen wichtigen und eigenständigen Aspekt der schizophrenen Erkrankung dar. Wie durch High-Risk-Studien belegt wurde, sind Defekte der Exekutivfunktionen bereits vor Manifestation der Krankheit nachweisbar. So wurden in der „Edingburgh-High-Risk-Study“ bei Kindern mit einem erhöhten Erkrankungsrisiko Gedächtnisdefizite und Beeinträchtigungen bei Aufgaben mit exekutiven Anteilen festgestellt (Byrne et al, 1999).

Auch bei kindlichen Schizophrenien wurde eine Beeinträchtigung exekutiver Funktionen gefunden. In der Studie von Rhinewine et al. (2005) wurden Kinder mit frühem Krankheitsbeginn (<18 Jahre) und sehr frühem Krankheitsbeginn (<13 Jahre) hinsichtlich kognitiver Leistungen untersucht und mit Kontrollprobanden verglichen. Die Ergebnisse zeigten deutliche kognitive Defizite der beiden Patienten-Gruppen, vor allem im Bereich der exekutiven Funktionen. Die Beeinträchtigungen exekutiver Funktionen scheinen zudem im Krankheitsverlauf verhältnismäßig stabil fortzubestehen. Rund et al. (2007) stellten über einen Zeitraum von drei Jahren bei 111 Patienten mit einer schizophrenen Erkrankung keine signifikante Veränderung der exekutiven Defizite fest. Dies entspricht den Befunden einer Review-Untersuchung von Frangou (2010). Die Autorin verweist darauf, dass bei schizophrenen Kindern eine altersabhängige Verbesserung kognitiver Funktionen ausbleibt bzw. diese langsamer verläuft im Vergleich zu gesunden Kindern und Jugendlichen, wodurch Diskrepanzen der kognitiven Leistungsfähigkeit erklärlich werden. Im Übrigen ist die Diskrepanz auch durch die bei schizophrenen Kindern und Jugendlichen vorhandene Erschwernis, neue Informationen zu aquirieren und problemlösungsrelevante kognitive Strategien zu entwickeln.

Die höheren kognitiven Leistungen sind an ein intaktes Funktionieren des Arbeitsgedächtnisses gebunden, das als kontrollierende und regulierende Instanz fungiert. Baddeley und Hitch (1974) bzw. Baddeley (2003) formulierten das Arbeitsgedächtnis als Drei-Komponenten-Modell mit einem Arbeitsspeicher für sprachliche und visuell-räumliche Informationen sowie einer weiteren Komponente für die exekutive Kontrolle. Es enkodiert, speichert und manipuliert vorübergehend relevante und nützliche Informationen, die dann höheren mentalen oder verhaltensrelevanten Prozessen zur Verfügung stehen. Zentraler Sitz des Arbeitsgedächtnisses ist der dorsolaterale präfrontale Cortex (DLPFC) mit seinen kortikalen und subkortikalen Verbindungen (s. Kap. 12.3 u. 12.7). Diese fronto-kortiko-subkortikalen Verschaltungen und Verknüpfungen mit multimodalen Assoziationsarealen bilden die Basis für die Integration unterschiedlicher Informationen, welche wiederum Voraussetzung für kohärente, zielgerichtete Handlungs- und Planungsvollzüge sind. Der präfrontale Kortex, speziell der DLPFC, hat eine große Bedeutung für die aktive Aufrechterhaltung von Informationen über ein bestimmtes Zeitintervall hinaus. Eine besondere Rolle scheinen dabei die Sustained-activity-Neurone im DLPFC zu spielen, die Informationen über eine Handlungstendenz auch gegen intermittierende Unterbrechungen der sensorischen Kontrolle aufrechterhalten (Fuster 1990; Goldman-Rakic und Selemon, 1997; Silver und Feldman, 2005). Es herrscht Konsens darüber, dass die Komplexität exekutiver Handlungskontrollen durch die koordinierte Aktivitäteines weiträumig verschalteten Netzwerkes von Hirnarealen mit besonderer Beteiligung frontaler Kortexregionen gewährleistet wird. 
Eine weitere für die Schizophrenie bedeutsame kognitive Beeinträchtigung betrifft den Transport von Informationen aus dem Arbeitsgedächtnis in das deklarative Gedächtnis. Bisherige ereigniskorrelierte fMRT-Studien fanden heraus, dass vor allem der linke PFC an der Verarbeitung und Enkodierung von semantischen Informationen und dem aktiven Abruf von Informationen aus dem Gedächtnis beteiligt ist. Neuere Studien konnten zudem zeigen, dass der mediale Temporallappen und insbesondere der in dessen Tiefe gelegene Hippocampus ebenfalls eine entscheidende Rolle bei Gedächtnisprozessen spielen (Wagner et al, 1998). Eine erfolgreiche Enkodierung von Informationen in das deklarative Gedächtnis wird nur durch die abhängige Zusammenarbeit von frontalen und mediotemporalen Hirnarealen ermöglicht (Fernandez und Tendolkar, 2001). Die frontalen Areale übernehmen hierbei eine vorgeschaltete organisierende Funktion, um die dauerhafte Überführung der zu speichernden Information durch den medialen Temporalkortex vorzubereiten.

Schizophrene Patienten zeigen verminderte kognitive Leistungen bei Aufgaben, die das Arbeitsgedächtnis beanspruchen. Während der Durchführung solcher Aufgaben ist die Aktivierung des DLPFC vermindert. Dies ist möglicherweise bedingt durch eine Beeinträchtigung GABAerg vermittelter inhibitorischer Prozesse im DLPFC. Denn: Sowohl die Synthese als auch die Wiederaufnahme freigesetzter GABA in die präsynaptische Nervenendigung, wo sie vor dem metabolischen Abbau geschützt ist, ist in den CABAergen Neuronen des DLPFC herabgesetzt (Lewis und Moghaddam 2006).

Infolge der GABA-Insuffizienz kommt es zu einer Aufhebung des inhibitorischen Einflusses auf die extrazelluläre Glutamatfreisetzung im DLPFC. Der dadurch bedingte Glutamatüberschuss hat wiederum exzitotoxische Auswirkungen auf die präfrontalen Neurone (s. Kap. 12.8). Die gleiche Situation (vermehrte kortikale Glutamatfreisetzung) kann auch durch die Applikation von NMDA-Rezeptorantagonisten herbeigeführt werden, wie dies u.a. durch fMRT-Studien belegt werden konnte (Jackson et al. 2004). Die Auswirkungen der CABAerg/glutamatergen Dysbalance zeigen sich klinisch in schizophrenietypischen kognitiven Dysfunktionen, speziell einer verminderten Effizienz des DLPFC und den entsprechenden Beeinträchtigungen des Arbeitsgedächtnisses.

Funktionsstörungen im Bereich des Arbeitsgedächtnisses manifestieren sich klinisch bei schizophrenen Patienten in den beschriebenen charakteristischen Denkstörungen, sowie in Beeinträchtigungen des zielorientierten und situationsadäquaten Verhaltens. Darüber hinaus führen sie als Folge desorganisierter kognitiver Prozesse zu schizophrenietypischen Defiziten der Handlungsplanung und Entscheidungsfindung.

Im Gegensatz zu produktiven psychotischen Symptomen sind kognitive Defizite bei Patienten mit einer schizophrenen Erkrankung bereits vor und während der Erstmanifestation nachweisbar (Bilder et al. 2000, Mayoral et al. 2008). Während produktive psychotische Symptome als „State-Marker“ gelten, zeigen kognitive Beeinträchtigungen Charakteristika eines so genannten „Trait-Markers“, das heißt, psychotische Symptome treten im Gegensatz zur kognitiven Symptomatik zustandsabhängig auf. Meist können kognitive Defizite im klinischen Alltag nur indirekt erschlossen werden, um genauere Angaben zu haben, muss eine neuropsychologische Untersuchung durchführt werden.

Es gibt eine Reihe von Varianten für die Untersuchung von Arbeitsgedächtnisleistungen. Ein Beispiel sind Aufgaben zur Zahlenspanne, die das Erinnern einer auditiv dar- 
gebotenen Zahlenreihe untersuchen, welche im nächsten Schritt rückwärts wiedergegeben werden soll. Dieses Testverfahren erfordert reine Gedächtnisleistungen, stellt aber auch Anforderungen an das Arbeitsgedächtnis, weil das Wiedergeben in umgekehrter Reihenfolge das kurzfristige Speichern und gleichzeitige Verarbeiten der wahrgenommenen Information voraussetzt. Ähnliche Anforderungen an das Arbeitsgedächtnis stellt auch der „Trail-Making-Test“ (TMT), der ebenfalls aus zwei Teilen besteht. Zu Beginn werden Zahlen (von 1 bis 25) präsentiert, die der Proband in aufsteigender Reihenfolge schnellstmöglich miteinander verbinden soll. Der zweite Teil beinhaltet dann zusätzlich zu den Zahlen (von 1 bis 13) auch Buchstaben (A bis L), die dann im Wechseln aufsteigend miteinander verbunden werden sollen (Bsp. 1-A-2-B etc.).

Mit den so genannten Tower-Tests, wie dem „Tower of Hanoi“ oder „Stockings of Cambridge“, wird das planende motorisch-räumliche Handeln untersucht. Den Probanden werden je nach Testvariante variierende Elemente präsentiert, die es zu sortieren gilt. Dabei werden durch bestimmte Restriktionen die Handlungsmöglichkeiten begrenzt, sodass mehrere Handlungsschritte bereits im Voraus geplant werden müssen, um das Ziel zu erreichen.

Einer der in Studien zu Schizophrenie am häufigsten verwendete Test ist der „Wisconsin Card Sorting Test“ (WCST), der von Berg (1948) zur Untersuchung der kognitiven Flexibilität bei Konzeptwechseln konzipiert wurde. Dem Probanden werden visuell Karten präsentiert, die unter unbekannten Zuordnungsregeln sortiert werden sollen. Während der Testdurchführung ändern sich die Regeln unangekündigt. Nur die Rückmeldung, ob die zuletzt präsentierte Karte korrekt sortiert wurde, bietet dem Probanden Hinweise auf Zuordnungsregeln .

Die kognitiven Beeinträchtigungen schizophrener Patienten können unterschiedliche Ausmaße erreichen. Bei etwa 20-25\% sind die Unterschiede im Vergleich zu Cesunden relativ gering ausgeprägt. Neuropsychologisch stärker beeinträchtigte Patienten weisen eine stärkere Tendenz zu negativen Symptomen und einem früheren Erkrankungsbeginn auf (Kremen et al. 200o). Darüber hinaus bestehen neuroanatomische Unterschiede:

Patienten mit geringen Einbußen ihrer kognitiven Fähigkeiten weisen eine allgemeine Abnahme der grauen Substanz und erweiterte dritte Ventrikel auf, während bei kognitiv stärker beeinträchtigten Patienten zusätzlich eine Abnahme der weißen Substanz und Erweiterungen der Seitenventrikel bestehen (Wexler et al. 2009).

\subsection{Soziale Kognitionen ${ }^{3}$}

Unter dem Begriff „Soziale Kognition“ versteht man allgemein die Art und Weise wie Menschen über sich selbst und ihr soziales Umfeld denken, oder spezifischer, wie sie soziale Informationen selektieren, interpretieren und im Gedächtnis behalten, um Entscheidungen zu treffen und Urteile zu bilden (Aronson et al. 2004). Soziale

3 Bei der Abfassung dieses Abschnitts war Frau Dipl.-Psych. Ch. Totzeck freundlicherweise behilflich. 
Kognitionen können in sozialen Interaktionen sowohl bewusst als auch unbewusst ablaufen. Sie umfassen neben dem Erkennen und Interpretieren von emotionalen Gesichtsausdrücken, Sprachmelodien (Prosodie) und Körperhaltungen auch das Differenzieren und Reflektieren eigener und fremder Absichten, Intentionen, Gedanken und Emotionen (Adolphs 2001, Brüne 2008). Letzteres geht auf Premack und Woodruff (1978) zurück und wird als „Theory of Mind“ bezeichnet: Die kognitive Fähigkeit, sich selbst und anderen mentale Zustände (mental states) - wie Gedanken, Absichten, Wünsche, Erwartungen und Vorstellungen - zuschreiben zu können, und somit in der Lage zu sein, ein Verhalten zu erfassen, zu erklären, zu beeinflussen und vorherzusagen.

Das intakte Funktionieren sozialer Kognitionen ermöglicht das Herstellen und Aufrechterhalten von Beziehungen zwischen sich und anderen, um in soziale Gruppen aufgenommen zu werden (Frith 2008) und das soziale Verhalten zu steuern (Couture et al. 2006, Sergi et al. 2006). Neuropsychologische fMRT-Studien befassen sich zurzeit vor allem mit den neuroanatomischen Substraten sozialer Kognitionen und erforschen spezifische neurale Strukturen, Gene und Neurotransmitter-Systeme, welchen bei der Wahrnehmung und Verarbeitung sozialer Informationen eine entscheidende Rolle zukommt. Nach Adolphs (2001) sind bei der Wahrnehmung sozial-relevanter Stimuli vor allem die kortikalen Regionen des Temporallappens beteiligt. Um eine Verbindung herzustellen zwischen den wahrgenommenen sozialen Informationen und einer adäquaten Reaktion wie bspw. einer Emotion, ist jedoch ein neurales Netzwerk bestehend aus den Amygdalae, dem präfrontalen, cingulären und dem somatosensorischen Cortex notwendig (Lee et al. 2004).

Beeinträchtigungen der sozialen Kognitionen werden bei Patienten mit einer schizophrenen Erkrankung zunehmend als allgemein gültige, klinisch relevante Merkmale anerkannt. In besonderem Maße sind hierbei die Wahrnehmung und Verarbeitung emotionaler Gesichtsausdrücke betroffen (Heimberg et al. 1992). Klinische Befunde deuten auf Leistungsdefizite schizophrener Patienten hin, mimisch dargebotene Emotionen korrekt zu benennen und von anderen Emotionen unterscheiden zu können (Grüsser et al. 1990, Schneider et al. 2008). Die beeinträchtige Leistung hängt mit dem Schweregrad der Symptomatik, insbesondere der Negativ-Symptomatik, zusammen (Kohler et al. 2000, 2003), und bezieht sich vornehmlich auf das Erkennen der Emotionen Angst und Ärger (Rasetti et al. 2009, Williams et al. 2007). Neue fMRTBefunde konzentrieren sich auf neuroanatomische Auffälligkeiten schizophrener Patienten während der Bewältigung von Aufgaben zur Diskrimination und Identifikation emotionaler Gesichtsausdrücke (Brunet-Gouet u. Decety 2006). Die Fähigkeit, visuell wahrgenommene emotionale Gesichtsausdrücke zu erkennen und zu deuten, ist vor allem im fusiformen Gyrus und dem Sulcus temporalis superior (STS) verankert (Haxby et al. 2000, Winston et al. 2004). Leitman et al. (2008) spezifizierten ihre fMRT-Untersuchung auf die Wahrnehmung ängstlicher Gesichter und fanden bei schizophrenen Patienten eine Störung der funktionellen neuralen Konnektivität zwischen Thalamus, Amygdala und präfrontalen Cortexarealen während der Durchführung von Aufgaben, bei denen mimische Ausdrucksformen von Angst und Bedrohung richtig erkannt und interpretiert werden sollten. Diese Befunde weisen darauf hin, dass die Beeinträchtigung der Fähigkeit, Angst in der Mimik eines Gegenübers wahrzunehmen und die damit zusammenhängende Bedrohung zu deuten, auf einer abnormalen Integration der wahrgenommenen sozialen Stimuli beruht. Dabei ist davon auszugehen, dass die Verarbeitung und Bewertung von Signalen der Bedrohung in- 
nerhalb der fronto-kortikalen Regionen und medialen Strukturen wie Amygdala und Thalamus unterbrochen wird und es dadurch zu einer Fehl-Interpretation der Situation und einer entsprechend inadäquaten Reaktion kommen kann. Emotionale Wahrnehmungsstörungen beschränken sich bei schizophrenen Erkrankungen nicht nur auf angstbezogene Stimuli sondern äußern sich in einer Beeinträchtigung, den Bedeutungsgehalt emotionaler und sozialer Signale der Außenwelt zu erfassen und korrekt $\mathrm{zu}$ interpretieren. Letzteres ist an das intakte Funktionieren des ventromedialen präfrontalen und orbitofrontalen Kortex gebunden. Neuroanatomische Läsionen in diesem Bereich führen zu stereotypen und sozial unangemessenen Verhaltensweisen (Adolphs 1999), welche auch meist bei schizophrenen Patienten zu beobachten sind.

Beeinträchtigungen der sozialen Kompetenz gehören zu den hervorstechendsten Merkmalen schizophrener Psychosen. Aus klinischer Sicht ist das inadäquate und oft bizarre Sozialverhalten der Patienten eines der charakteristischen Merkmale der Schizophrenie (Brüne 2005).

Beeinträchtigungen der sozialen Kompetenz sind bei schizophrenen Störungen im gesamten Verlauf der Erkrankung festzustellen. Bereits vor Beginn der psychotischen Kernsymptomatik zeigen sich sozial-kognitive Defizite, die sich im weiteren Verlauf meist verschlechtern (Davidson et al. 1999). Zudem scheinen die fehlenden sozialen Kompetenzen zu der Rezidivrate beizutragen (Perlick et al. 1992; Pinkham et al. 2003).

Eine Möglichkeit der testpsychologischen Untersuchung der ToM bieten Aufgaben zur Mentalisierungsfähigkeit, die in experimentell konstruierten Situationen das Reflektieren einer falschen Überzeugung anderer Personen über eine Handlung (Metarepräsentationen) erfordern, so genannte "first order false belief“ Tests. Pilowsky et al. (2000) konnten in einer vergleichenden Studie mit 12-jährigen Kindern zeigen, dass sich schizophrene Kinder tatsächlich eher von eigenen Vorstellungen leiten ließen und den Kenntnisstand der handelnden Person ignorierten, was zu einer deutlich erhöhten False-belief-Fehlerrate führte. Komplexer wird die ToM-Testung in „second order false belief“- oder „Faux pas“(FP)-Tests, die Annahmen über eine weitere Person innerhalb derselben konstruierten Situation erfragen und damit eine höhere kognitive Leistung erfordern. In der Untersuchung von Herold et al. (2008) erbrachten Patienten bereits in der frühen Erkrankungsphase einer schizophrenen Störung (erste Symptome lagen weniger als 5 Jahre zurück) signifikant schlechtere Leistungen in FP-Tests. Die zusätzlich durchgeführte Voxel-basierte Morphometrie (VBM) ergab in der Patientengruppe eine signifikant verringerte Dichte der Grauen Substanz in bestimmten frontalen, temporalen und subkortikalen Hirnregionen. Die schwächere FP-Leistung der schizophrenen Patienten korrelierte mit den Reduktionen der Grauen Substanz im linken orbitofrontalen Cortex und dem rechten Temporallappen. Diese Befunde führen zu der Schlussfolgerung, dass bei Erkrankungsbeginn die herabgesetzten ToM-Fähigkeiten von schizophrenen Patienten mit Reduktionen der Grauen Substanz im linken orbitofrontalen Cortex und rechten Temporallappen assoziiert sein könnten (Herold et al. 2008).

Bisher konnte jedoch noch nicht eindeutig erwiesen werden, inwieweit die falschen Schlussfolgerungen schizophrener Patienten bei den bildlich dargebotenen Stimuli der False belief- und Fauxpas-Aufgaben auch durch eine fehlerhafte Wahrnehmung von visuellen Bewegungsabläufen entstehen können (Hooker \& Park 200o). Diesen Einwand berücksichtigend führten Schiffman et al. (2004) eine weitere Untersuchung 
der ToM bei schizophrenen Kindern durch. In einer Langzeitstudie sollten Kinder mit familiärer genetischer Prädisposition für die Entwicklung einer Schizophrenie in einem so genannten Role Taking Test ihre Fähigkeit unter Beweis stellen, fremde Rollenmuster einnehmen zu können. Die Kinder, die 20 Jahre später an einer Schizophrenie erkrankten, erzielten tatsächlich deutlich schlechtere Test-Ergebnisse. Die fehlerhafte Zuschreibung innerer Zustände von fremden Personen erklärt Frith (1992, 2004) in seinem kognitionspsychologischen Modell als ein Defizit der Metarepräsentation bei Schizophrenen. Frith unterscheidet in seinem Modell drei Störungsmuster, die entsprechend unterschiedliche Symptomkonstellationen bedingen. Zum Einen ist bei schizophrenen Patienten eine Störung des Handelns aufgrund eigener Willensbildung (,willed action“) erkennbar, die das Umsetzen eigener Intentionen in Handlungen und das Generieren von spontanem Verhalten erschwert. Dies kann sich klinisch in negativen Symptomen und desorganisiertem Verhalten der Patienten äußern. Des Weiteren kann eine Störung der Selbststeuerung („self-monitoring“) vorliegen, die erklärt, warum sich Patienten als fremdgesteuert empfinden und Ich-Störungen (wie bspw. Gedankeneingebung und Gedankenausbreitung) auftreten. Die dritte Form bezeichnet Frith als Störung des Reflektierens der Intentionen Anderer („monitoring the intentions of others“). Schizophrene Patienten weisen meist Defizite auf, die Intentionen und Absichten Anderer korrekt zu bewerten und darauf adäquat zu reagieren, was sich zum Beispiel in Verfolgungs- und Beziehungswahn bemerkbar machen kann (siehe auch Brüne 2008).

Zusammenfassend lässt sich sagen, dass die stärkere Beachtung sozial-kognitiver Prozesse das Verständnis der schizophrenen Kernsymptomatik fördert. Insbesondere für die nicht-medikamentösen Therapiestrategien spielen die bei schizophrenen Erkrankungen nachgewiesenen sozial-kognitiven Störungen eine Schlüsselrolle, die bereits in der Prävention psychotischer Störungen erfasst und berücksichtigt werden sollten (s. Kap. 13.3). 